\title{
Vector-Sensor MUSIC for Polarized Seismic Sources Localization
}

\author{
Sebastian Miron \\ Laboratoire des Images et des Signaux (LIS), 961 rue de la Houille Blanche, BP 46, 38402 Saint Martin d'Hères Cedex, France \\ Email: sebastian.miron@lis.inpg.fr
}

Nicolas Le Bihan

Laboratoire des Images et des Signaux (LIS), 961 rue de la Houille Blanche, BP 46, 38402 Saint Martin d'Hères Cedex, France Email: nicolas.le-bihan@lis.inpg.fr

\begin{abstract}
Jérôme I. Mars
Laboratoire des Images et des Signaux (LIS), 961 rue de la Houille Blanche, BP 46, 38402 Saint Martin d'Hères Cedex, France Email: jerome.mars@lis.inpg.fr
\end{abstract}

Received 18 December 2003; Revised 7 June 2004

\begin{abstract}
This paper addresses the problem of high-resolution polarized source detection and introduces a new eigenstructure-based algorithm that yields direction of arrival (DOA) and polarization estimates using a vector-sensor (or multicomponent-sensor) array. This method is based on separation of the observation space into signal and noise subspaces using fourth-order tensor decomposition. In geophysics, in particular for reservoir acquisition and monitoring, a set of $N_{x}$-multicomponent sensors is laid on the ground with constant distance $\Delta x$ between them. Such a data acquisition scheme has intrinsically three modes: time, distance, and components. The proposed method needs multilinear algebra in order to preserve data structure and avoid reorganization. The data is thus stored in tridimensional arrays rather than matrices. Higher-order eigenvalue decomposition (HOEVD) for fourthorder tensors is considered to achieve subspaces estimation and to compute the eigenelements. We propose a tensorial version of the MUSIC algorithm for a vector-sensor array allowing a joint estimation of DOA and signal polarization estimation. Performances of the proposed algorithm are evaluated.
\end{abstract}

Keywords and phrases: vector-sensor array, vector MUSIC, interspectral tensor, higher-order eigenvalue decomposition for 4thorder tensors.

\section{INTRODUCTION}

Seismic measurements are used for mapping geological features to discover, locate, and evaluate gas concentrations or oil reservoirs. For this purpose, geophysicists study elastic waves propagating in the earth originating from artificial sources (such as explosions) and recorded on a sensor array (Figure 1). To analyze the recorded data, models of waveforms are used and estimation techniques are applied to find parameters describing the waves such as their direction of arrival (DOA), polarization, power, and so forth. From the estimated parameters, it is possible to obtain information on layer structure, depth, and so forth. [1]. In order to map a field or a reservoir, a sensor array which gives a 2D signal $s\left(t_{n}, x_{n}\right)$ of size $N_{x} \times N_{t}$ is generally used $\left(t_{n}\right.$ is the time recording dimension and $x_{n}$ is the distance dimension (array aperture)). After performing a Fourier transform along the time dimension, classical scalar-sensor version of the MUSIC algorithm can be used in order to estimate the DOAs of sources [2].

Vector sensors are nowadays widely used in seismic acquisition allowing a better characterization of the layers because of the polarization dimension added to detection process. One sensor measures the particle-displacement vector that describes the particle motion in $3 \mathrm{D}$ at a given point in space. As the polarization is wavefield dependent, it is used as an essential attribute to separate waves in addition to their different DOAs. In the last decade, many arrayprocessing techniques for source localization and polarization estimation using vector sensors have been developed, mainly in electromagnetics. Nehorai and Paldi [3] developed the Cramer-Rao bound (CRB) for this problem and the vector crossproduct DOA. Li and Compton [4] developed the ESPRIT algorithm for a vector-sensor array. MUSIC-based algorithms for this problem were also proposed by Wong and Zoltowski $[5,6,7]$, who also developed vector-sensors 


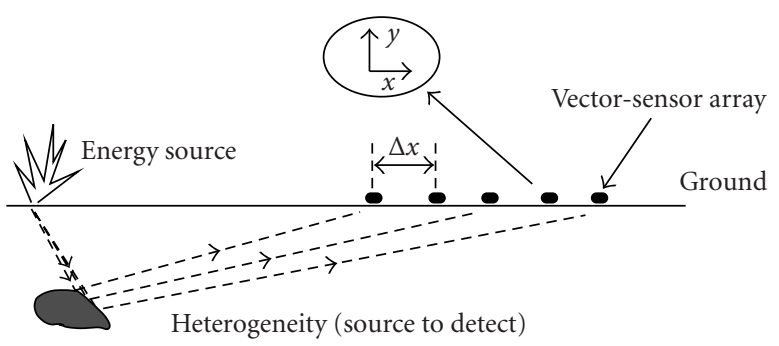

FIgURe 1: Seismic acquisition scheme (reflection).

versions of ESPRIT $[8,9,10,11,12]$. These approaches use matrix techniques directly derived from scalar-sensor array processing. Such a method is based on the long-vector approach, consisting in the concatenation of all components of the vector-sensor array in a long vector of size $N_{c} N_{x}$ [8]. The originality of our method consists in keeping multidimensional structures for data organization and processing. These structures are more adapted to the nature of polarized signals, allowing data organization closer to its multimodal intrinsic nature. A block of acquisition data is represented as a $3 \mathrm{D}$ table of size $N_{c} \times N_{x} \times N_{t}$, where $N_{c}$ is the number of components of each vector sensor, $N_{x}$ is the array aperture (number of sensors), and $N_{t}$ is the number of time samples. The proposed method is a version of MUSIC algorithm adapted to this multilinear structure. The technique is very efficient for geophysicists purpose where seismic sources have a strong nonstationary behavior.

This correspondence is organized as follows. In Section 2, a brief presentation of scalar-sensor array MUSIC algorithm is given. The vector version of this estimator is then developed in Section 3. The performance of the proposed algorithm evaluated in simulations is described in Section 4.

\section{SCALAR-SENSOR MUSIC}

In order to better understand the multicomponent approach, a short reminder of scalar-sensor MUSIC technique is presented.

The main assumptions used for the scalar-sensor MUSIC algorithm are listed below.

(A1) Consider the case of a linear, uniform scalar-sensor array.

(A2) The recorded signals are far-field seismic waves propagated in an isotropic, homogeneous medium.

(A3) The signal is made of $K$ sources ( $K$ is known). If $K$ is not known, it can be estimated from the eigenvalues variation [13].

(A4) Sources $\left(s_{1}, s_{2}, \ldots, s_{K}\right)$ are statistically decorrelated and spatially coherent.

(A5) They are all confined in the array plane.

(A6) Sources are considered as centered unknown deterministic processes.

(A7) There are more sensors than sources $\left(N_{x}>K\right)$.

(A8) The noise is centered and spatially white.

\subsection{Scalar-sensor array model}

If we choose the first sensor of the array as a reference, the output of the $m$ th sensor in frequency domain is given by

$$
X_{m}(\nu)=\sum_{k=1}^{K} S_{k}(\nu) \exp \left(-j 2 \pi v \tau_{m k}\right)+N_{m}(\nu),
$$

where $S_{k}(\nu)$ is the complex amplitude of the $k$ th source, $N_{m}(\nu)$ is the additive noise on sensor $m$, and $\tau_{m k}$ is the propagation delay between the reference and the $m$ th sensor for source $k$. Intersensor phase-shift $\theta_{k}$, corresponding to the $k$ th source will be used to characterize the DOA of the source on the array rather than the incidence angle $\alpha_{k}$ (because it is independent of physical quantities such as intersensors distance $\Delta x$ and wave propagation velocity $v_{k}$ ). The incidence angle $\alpha_{k}$ can then be calculated using the following relation:

$$
\theta_{k}=2 \pi \nu \frac{\Delta x \sin \alpha_{k}}{v_{k}} .
$$

The array output at frequency $v$ is a vector containing the sensors samples as

$$
\mathbf{x}(\nu)=\left[X_{1}(\nu) \cdots X_{N_{x}}(\nu)\right]^{T},
$$

where $T$ is the matrix transposition operator. For a plane wave impinging on an antenna, the source vector $\mathbf{a}_{k}$ models $k$ th-source propagation on the antenna and has the following expression:

$$
\mathbf{a}_{k}\left(\theta_{k}\right)=\frac{1}{\sqrt{N_{x}}}\left[1, e^{-j \theta_{k}}, \ldots, e^{-j\left(N_{x}-1\right) \theta_{k}}\right]^{T},
$$

where $\theta_{k}$ is the intersensor phase-shift corresponding to the source $S_{k}$.

\subsection{Spectral matrix and scalar-MUSIC estimator}

The interspectral matrix (or crossspectral matrix) defines second-order statistical relationships between the array outputs at a given frequency [14]. This matrix is described as

$$
\widehat{\Gamma}=\xi\left\{\mathbf{x x}^{H}\right\},
$$

where $H$ is the complex conjugate transpose and $\xi\{\cdot\}$ is the mathematical expectation operator which allows to access an estimate of the interspectral matrix $\hat{\Gamma}$. Without the $\xi\{\cdot\}$ operator (smoothing operator), $\Gamma$ is a rank-one matrix (any decomposition can be found and sources are fully correlated). Eigenstructure-based source localization techniques, such as ESPRIT and MUSIC, assume nonsingular signal correlation matrix. In geophysical prospection, because of the impulsive nature of sources, the recorded signals have a strong nonstationary behavior. In order to decorrelate the signals in the interspectral matrix, several smoothing techniques have been proposed $[15,16,17,18]$, mainly based on the spectral and spatial diversity of the signals. The drawback of spatial smoothing is the reduction of effective array-aperture length, resulting in lower resolution and accuracy, while the frequency averaging induces bias in the DOA estimates. 
The eigenvalue decomposition (EVD) of interspectral matrix yields

$$
\widehat{\boldsymbol{\Gamma}}=\sum_{i=1}^{N_{x}} \lambda_{i} \mathbf{v}_{i} \mathbf{v}_{i}^{H}
$$

with $\lambda_{1} \geqslant \lambda_{2} \geqslant \cdots \geqslant \lambda_{N_{x}} \geqslant 0$ being the eigenvalues and $\mathbf{v}_{i}$ the eigenvectors of $\hat{\boldsymbol{\Gamma}}$. The first $K$ eigenvectors are used to build the signal subspace projector such as

$$
\Pi_{S}=\sum_{i=1}^{K} \mathbf{v}_{i} \mathbf{v}_{i}^{H} .
$$

The noise subspace projector is then given by

$$
\boldsymbol{\Pi}_{N}=\sum_{i=K+1}^{N_{x}} \mathbf{v}_{i} \mathbf{v}_{i}^{H} .
$$

Scalar-MUSIC algorithm consists in estimating DOA by projection of the steering vector

$$
\mathbf{m}(\theta)=\frac{1}{\sqrt{N_{x}}}\left[1, e^{-j \theta}, \ldots, e^{-j\left(N_{x}-1\right) \theta}\right]^{T}
$$

on noise subspace. The estimator of DOA is obtained as the inverse of the Frobenius norm ${ }^{1}$ of this projection:

$$
\operatorname{MUSIC}(\theta)=\frac{1}{\left\|\boldsymbol{\Pi}_{N} \mathbf{m}(\theta)\right\|} .
$$

Scalar MUSIC yields asymptotically efficient estimation in case of uncorrelated or partially correlated signals.

Extended versions of MUSIC algorithm for multicomponent sensors (mainly developed for electromagnetic antennas) were proposed a couple of years ago, based on longvector approaches. In seismic prospection, the vector-sensor data processing is most often done by applying the classical MUSIC algorithm independently on each component. In order to take into account the coherent information between the components, we propose a version of the algorithm adapted to the multidimensional nature of seismic vectorsensor arrays acquisitions, for a better DOA estimation.

\section{VECTOR-SENSOR ARRAY PROCESSING}

\subsection{Polarization and vector signal}

We start with considering the general case of a polarized signal in a 3D space. Suppose we have a vector sensor at the origin of a spherical coordinate system, as shown in Figure 2. One can show that for a seismic wave propagating in a $3 \mathrm{D}$ medium, particle movements are confined in a plane (polarization plane). Assume that a signal is arriving from direction $(\alpha, \beta)$, where $\beta \in[-\pi / 2, \pi / 2]$ is the elevation angle and

\footnotetext{
${ }^{1}$ The Frobenius norm of a vector $\mathbf{v}$ is given by $\|\mathbf{v}\|=\sqrt{\langle\mathbf{v}, \mathbf{v}\rangle}$, where $\langle\cdot\rangle$ is a classical scalar product.
}

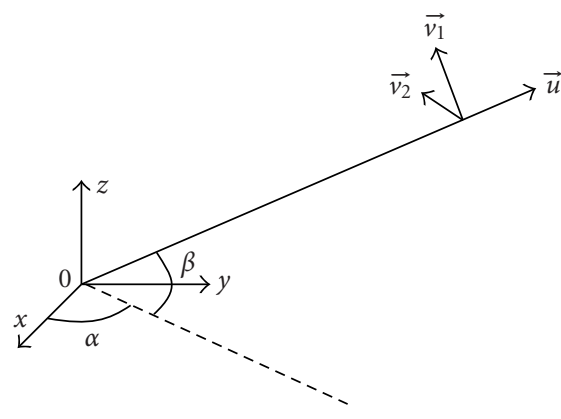

FIGURE 2: Vector-sensor array coordinate system.

$\alpha \in[-\pi, \pi]$ is the azimuth angle. We consider the polarization ellipse of the source in a fixed transverse plane spanned by vectors $\vec{v}_{1}, \vec{v}_{2}$. The unit vectors $\vec{v}_{1}, \vec{v}_{2}$, and $\vec{u}$ (in that order) form a right-handed coordinate system $(-\vec{u}$ is a unit vector pointing in the signal propagation direction). The coordinates of $\vec{u}$ in the sensor's $(x, y, z)$ coordinate system can be written as

$$
\overrightarrow{\mathbf{u}}=\left[\begin{array}{lll}
\cos \alpha \cos \beta & \sin \alpha \cos \beta & \sin \beta
\end{array}\right]\left[\begin{array}{l}
\vec{x} \\
\vec{y} \\
\vec{z}
\end{array}\right] .
$$

In relation (11),

$$
\mathbf{u}=\left[\begin{array}{lll}
\cos \alpha \cos \beta & \sin \alpha \cos \beta & \sin \beta
\end{array}\right]^{T}
$$

represents the coordinates of the propagation vector in the sensor coordinates system. An incoming wave $s(t)$ generates three signals on the three outputs $s_{x}(t), s_{y}(t)$, and $s_{z}(t)$ of the vector sensor. The vector-sensor response model to $s(t)$ can thus be written as

$$
\mathbf{s}(t)=\left(\begin{array}{l}
s_{x}(t) \\
s_{y}(t) \\
s_{z}(t)
\end{array}\right)=\mathbf{P u} w(t)
$$

with $w(t)$ the complex signal of the source signal $s(t)$ and $\mathbf{P}$ a matrix describing the orientation angle of the polarization plane and the parameters of polarization ellipse. $\mathbf{P}$ has different expressions for different types of seismic waves [19] (compressional, shear, Rayleigh, Love, etc. [1]).

In this paper, we propose a tensorial model for a polarized source arriving on a vector-sensor array. This model contains the same statistical information as the long-vector model plus a modal (orientation) information (distancecomponents). A tensor can be seen as a higher-ordered ( $>2)$ dimensional table of numerical values with respect to a number of bases. The real or complex-valued tensors of a given dimensionality form a linear vector space which implies definition of sum and multiplication with a scalar (we will consider the componentwise sum and scalar multiplication).

In order to determine the transfer function of a vectorsensor array to an incoming polarized source, more assumptions have to be done in addition to those describing the scalar case. 
(A9) We suppose that the additive noise on the sensors is not polarized (the crosscovariance matrix for noise on a vector-sensor components is diagonal).

(A10) Sources polarization is constant in time and along the antenna (temporal and spatial stationarity).

(A11) Sources have different polarizations.

The mathematical model of a source $k$ can be written as the outer product of a vector containing information on the source behavior along the antenna $\mathbf{a}_{k}$, and a polarization vector $\mathbf{p}_{k}$ yielding the energy repartition between the components of the vector sensor:

$$
\mathbf{A}_{k}=\mathbf{a}_{k} \circ \mathbf{p}_{k} .
$$

The outer product $\mathcal{A} \circ \mathscr{B}$ of a tensor $\mathcal{A} \in \mathbb{C}^{I_{1} \times I_{2}}$ and a tensor $\mathscr{B} \in \mathbb{C}^{I_{1} \times J_{2}}$ is defined by

$$
(\mathcal{A} \circ \mathscr{B})_{i_{1} i_{2} j_{1} j_{2}}=a_{i_{1} i_{2}} b_{j_{1} j_{2}}
$$

for all values of indices.

The polarized wave model described before assumes three components oriented in the three-physical-space directions. In seismic prospection, this is not always the case. Seismic vector sensors are composed of two to four components, geophones (recording ground movements), and hydrophones (water pressure sensors, for ocean bottom investigations). In this case, the geometrical orthogonality between components is no longer respected. This is why we propose a polarization model less complex but more flexible, fitting with the acquisition system. The DOA of the source $k$ will thus be characterized only by the azimuth angle $\alpha_{k}$ (or the corresponding intersensor phase-shift quantity $\theta_{k}$ ). We consider the case of a two-component $(2 \mathrm{C})\left(N_{c}=2\right)$ vectorsensor array (the formalism remains the same in the case of an arbitrary $N_{c}$ ). The polarization vector for the $k$ th source can thus be written as

$$
\mathbf{p}_{k}\left(\rho_{k}, \varphi_{k}\right)=\frac{1}{\sqrt{\rho_{k}^{2}+1}}\left[1, \rho_{k} e^{j \varphi_{k}}\right]
$$

in which $\rho_{k}$ and $\varphi_{k}$ are the amplitude ratio and the phase shift, respectively, between the second component of the sensor and the first. The behavior of a polarized source on a twocomponent vector-sensor array can be modeled by a secondorder tensor (a matrix) of rank 1:

$$
\mathbf{A}_{k}\left(\theta_{k}, \rho_{k}, \varphi_{k}\right)=\mathbf{a}_{k}\left(\theta_{k}\right) \circ \mathbf{p}_{k}\left(\rho_{k}, \varphi_{k}\right),
$$

where $\mathbf{p}_{k}$ is given by (16) and $\mathbf{a}_{\mathbf{k}}$ is such that

$$
\mathbf{a}_{k}\left(\theta_{k}\right)=\frac{1}{\sqrt{N_{x}}}\left[1, e^{-j \theta_{k}}, \ldots, e^{-j\left(N_{x}-1\right) \theta_{k}}\right]^{T} .
$$

We have to estimate $\theta_{k}, \rho_{k}$, and $\varphi_{k}$ to characterize a source on an $N_{c}=2 \mathrm{C}$ vector-sensor array. In frequency domain, outputs of the vector-sensor array are given by a $N_{x} \times N_{c}$ matrix in which every column corresponds to a vector component and each row to a sensor as follows:

$$
\mathbf{X}(\nu)=\left(\begin{array}{cc}
x_{11}(\nu) & x_{12}(\nu) \\
\vdots & \vdots \\
x_{N_{x} 1}(\nu) & x_{N_{x} 2}(\nu)
\end{array}\right) .
$$

If assumptions (A10) and (A11) are considered, at a given frequency $\nu_{0}, \mathbf{X}\left(\nu_{0}\right)$ can be written as a linear combination of $K$ unknown deterministic signals $s_{k}$ with additive white noise. For simplification, argument $v_{0}$ will be omitted so that $\mathbf{X}\left(\nu_{0}\right)=\mathbf{X}:$

$$
\left(\begin{array}{cc}
x_{11} & x_{12} \\
\vdots & \vdots \\
x_{N_{x} 1} & x_{N_{x} 2}
\end{array}\right)=\mathcal{A} \cdot 2\left(\begin{array}{c}
s_{1} \\
\vdots \\
s_{K}
\end{array}\right)^{T}+\left(\begin{array}{cc}
n_{11} & n_{12} \\
\vdots & \vdots \\
n_{N_{x} 1} & n_{N_{x} 2}
\end{array}\right) .
$$

The operator $\bullet_{2}$ represents the second-mode product of a tensor by a matrix (given by (21)).

The 2-mode product of a tensor $\mathcal{A} \in \mathbb{C}^{I_{1} \times I_{2} \times I_{3}}$ and a matrix $\mathbf{U} \in \mathbb{C}^{I_{2} \times I_{2}}$, denoted by $\mathscr{A}_{\bullet} \bullet_{2} \mathbf{U}$, is an $\left(I_{1} \times J_{2} \times I_{3}\right)$ tensor given by

$$
(\mathcal{A} \cdot 2 \mathbf{U})_{i_{1} j_{2} i_{3}}=\sum_{i_{2}} a_{i_{1} i_{2} i_{3}} u_{j_{2} i_{2}} .
$$

$\mathcal{A}$ is a third-order tensor regrouping all the information of the sources behavior on the vector-sensor array. Another way of writing (20) is

$$
\mathbf{X}=\sum_{k=1}^{K} \mathbf{A}_{k} s_{k}+\mathbf{N}
$$

with $\mathbf{N}$ the additive noise matrix.

In order to characterize the incident field, second-order statistics are considered through a "spectral tensor."

\subsection{Interspectral tensor}

Interspectral tensor is a fourth-order complex tensor of size $N_{x} \times N_{c} \times N_{x} \times N_{c}$ defined as the second-order automoments and crossmoments between all the components on all sensors as follows:

$$
\mathcal{T}=\xi\left\{\mathbf{X} \circ \mathbf{X}^{*}\right\},
$$

where an element of $\mathcal{T}$ is given by

$$
t_{i_{1} i_{2} i_{3} i_{4}}=\xi\left\{x_{i_{1} i_{2}} x_{i_{3} i_{4}}^{*}\right\} .
$$

If we replace (22) in (23) and using (A4), (A7), (A9), and (A10), $\mathcal{T}$ can be written as

$$
\mathcal{T}=\sum_{k=1}^{K} \sigma_{k}^{2} \mathbf{A}_{\mathbf{k}} \circ \mathbf{A}_{\mathbf{k}}^{*}+\mathcal{N},
$$

in which $\sigma_{k}^{2}$ represents the $s_{k}$-source power and $\mathcal{N}=\xi\{\mathbf{N}$ 。 $\left.\mathbf{N}^{*}\right\}$ is a fourth-order tensor containing the second-order noise statistics. 


\subsection{Higher-order eigenvalue decomposition}

Compared to the long-vector spectral matrix, spectral-tensor also includes an orientation (modal) information (distancecomponents), allowing a couple of different tensor decompositions corresponding to different types of tensor orthogonality [20]. The HOEVD studied in this paper uses the concept of simple orthogonality as it was also defined in [21]. HOEVD does not make full use of the modal information but it allows detection of $N_{x} N_{c}-1$ sources (almost $N_{c}$ times more than in the scalar case). Higher-order singular value decomposition (HOSVD) [21] improves the resolution power by imposing a more restrictive constraint, the strong orthogonality, forcing orthogonality along each mode in the spectral tensor. The main drawback of this method is that it allows detection of maximum $\min \left(N_{c}-1, N_{x}-1\right)$ sources (that is one source on a $2 \mathrm{C}$ sensor-array). This explains the choice of $\mathrm{HO}-$ EVD to illustrate tensor formalism in this paper. PARAFAC (parallel factors) decomposition [21] can also be applied to spectral tensor yielding an accurate tensor-rank estimation (a good estimator of source number). The drawback of this decomposition is that the resulting rank-1 tensors are not necessary orthogonal which is not suitable for decomposition in orthogonal subspaces. A detailed comparison of these tensor decompositions is beyond the scope of this paper and should be included in a subsequent paper.

As interspectral tensor presents a higher-order Hermitian symmetry $\left(t_{i_{1} i_{2} i_{3} i_{4}}=t_{i_{3} i_{4} i_{1} i_{2}}^{*}\right)$, De Lathauwer [21] shows that it can be decomposed into eigenelements by means of HOEVD. Interspectral tensor can be written as

$$
\mathcal{T}=\sum_{p=1}^{P} \lambda_{p} \mathbf{U}_{\mathbf{p}} \circ \mathbf{U}_{\mathbf{p}}^{*},
$$

where $P=N_{x} N_{c}, \lambda_{p}$ are real eigenvalues, and $\mathbf{U}_{\mathbf{p}} \in \mathbb{C}^{N_{x} \times N_{c}}$ are $P$ mutually orthonormal eigentensors. In order to define the mutually orthonormal eigentensors, it is necessary to generalize the well-known definitions of scalar product and orthogonality from vector case (first-order tensors) to second-order tensors. The scalar product $\langle\mathscr{A}, \mathscr{B}\rangle$ of two tensors $\mathcal{A}, \mathcal{B} \in \mathbb{C}^{I_{1} \times I_{2}}$ is defined as

$$
\langle\mathcal{A}, \mathcal{B}\rangle=\sum_{i_{1}, i_{2}} b_{i_{1} i_{2}}^{*} a_{i_{1} i_{2}} .
$$

Tensors of which the scalar product equals 0 are mutually orthogonal [21]. This definition is equivalent to simple orthogonality [20] and implies a global orthogonality of tensors.

To obtain the HOEVD of interspectral tensor, we apply the EVD on standard matrix unfoldings [21]. For a forthorder tensor $\mathcal{T} \in \mathbb{C}^{N_{x} \times N_{c} \times N_{x} \times N_{c}}$, there are four different ways to unfold it in order to obtain a square matrix. In our particular case, $t_{i_{1} i_{2} i_{3} i_{4}}=t_{i_{3} i_{4} i_{1} i_{2}}^{*}$ only two of these unfolding techniques yield Hermitian symmetric matrices. So, two linear mappings between the vector space of $\mathcal{T}$-like tensors and the vector space of Hermitian matrices $\mathbf{G}$ of size $N_{x} N_{c} \times N_{x} N_{c}$ can be defined as follows:

$$
\begin{aligned}
& g 1_{\left(\left(i_{2}-1\right) N_{x}+i_{1},\left(i_{4}-1\right) N_{x}+i_{3}\right)}=t_{\left(i_{1}, i_{2}, i_{3}, i_{4}\right)}, \\
& g 2_{\left(\left(i_{1}-1\right) N_{c}+i_{2},\left(i_{3}-1\right) N_{c}+i_{4}\right)}=t_{\left(i_{1}, i_{2}, i_{3}, i_{4}\right)}
\end{aligned}
$$

for all indices $i_{1}, i_{2}, i_{3}$, and $i_{4}$. In these expressions, $g_{(i, j)}$ are the entries of $\mathbf{G}$. The two decompositions $g 1$ and $g 2$ are equivalent, yielding the same eigentensors with HOEVD. This is a natural result, the HOEVD of a pairwise symmetric tensor being unique. The second-order eigentensors are obtained by reintroduction of the tensor notation for the resulting matrices in the decomposition (the inverse operation of matrix unfolding).

\subsection{Vector-MUSIC estimator}

By identification of (25) with (26), we associate the first $K$ eigenvalues to the signal part of the observation and the other $P-K$ eigenvalues to the noise part. We build the noise subspace projector using the last $P-K$ eigentensors as follows:

$$
\mathcal{P}_{\mathcal{N}}=\sum_{p=K+1}^{P} \mathbf{U}_{\mathbf{p}} \circ \mathbf{U}_{\mathbf{p}}^{*}
$$

The steering tensor $\mathbf{M}(\theta, \rho, \varphi)$ is generated as follows:

$$
\begin{aligned}
\mathbf{M}(\theta, \rho, \varphi) & =\mathbf{a}(\theta) \circ \mathbf{p}(\rho, \varphi) \\
& =\frac{1}{\sqrt{N_{x}\left(1+\rho^{2}\right)}}\left[\begin{array}{cc}
1 & \rho e^{j \varphi} \\
e^{-j \theta} & \rho e^{j(\varphi-\theta)} \\
\vdots & \vdots \\
e^{-j\left(N_{x}-1\right) \theta} & \rho e^{j\left(\varphi-\left(N_{x}-1\right) \theta\right)}
\end{array}\right] .
\end{aligned}
$$

Vector-MUSIC estimator, denoted VM, is then computed by projecting the steering tensor $\mathbf{M}$ on the noise subspace as follows:

$$
\operatorname{VM}(\theta, \rho, \varphi)=\frac{1}{\left\|\left\langle\mathcal{P}_{\mathcal{N}}, \mathbf{M}(\theta, \rho, \varphi)\right\rangle_{i_{1} i_{2}}\right\|}
$$

in which $\left\langle\mathcal{P}_{\mathcal{N}}, \mathbf{M}\right\rangle_{i_{1} i_{2}}$ is the inner product over the first two indices, and $\|\cdot\|$ is the Frobenius norm. ${ }^{2}$

MUSIC-estimator values are stored in a third-order multidimensional table. So, we can detect $N_{x} N_{c}-1$ sources, while in monocomponent-sensor case, we can estimate a maximum of $N_{x}-1$ DOAs. In the general case of an arbitrary number $N_{c}$ of components, the number of parameters to estimate is $2 N_{c}-1$. In the following section, this algorithm is evaluated on synthetic data in order to characterize and separate seismic sources in DOA-polarization domain.

\section{SIMULATION RESULTS}

To illustrate the performances of vector-MUSIC algorithm, we have considered a scenario with two seismic sources recorded on a $2 \mathrm{C}$ vector-sensor array. The polarization

\footnotetext{
${ }^{2}$ The Frobenius norm of a matrix $\mathbf{M}$ is given by $\|\mathbf{M}\|=\sqrt{\operatorname{Tr}\left(\mathbf{M M}^{H}\right)}$, where $\operatorname{Tr}(\cdot)$ is the trace of a square matrix.
} 


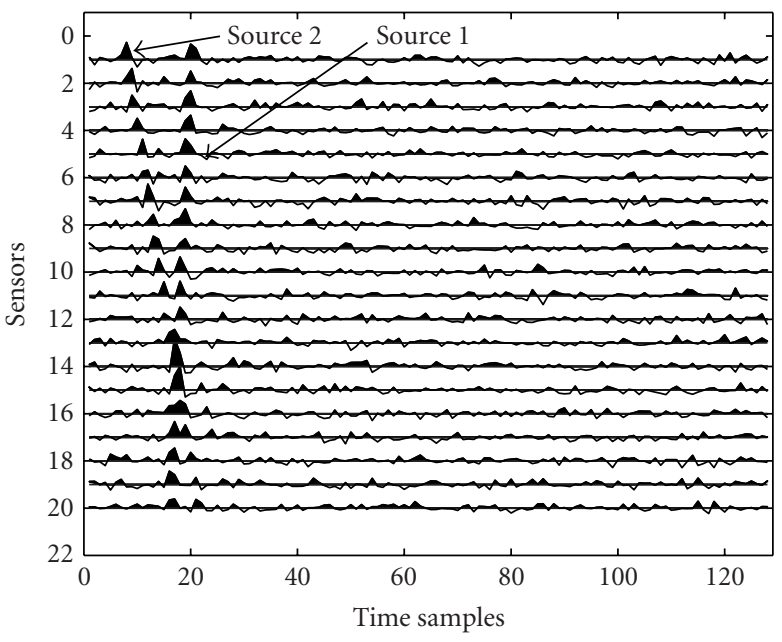

(a)

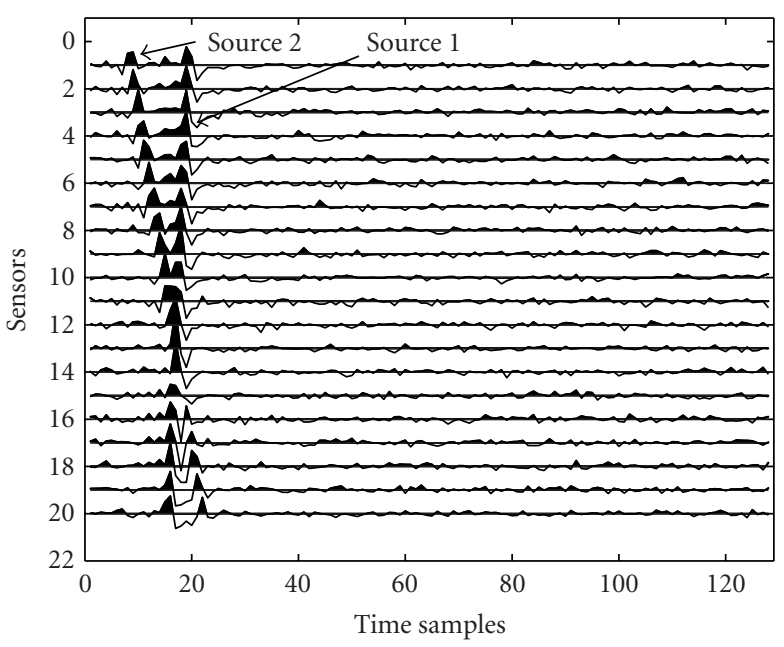

(b)

Figure 3: Two-component seismic section. (a) First component and (b) second component.

parameters simulated are $\rho_{1}=2, \varphi_{1}=-80^{\circ}(-1.4 \mathrm{rad})$ for the first source, and $\rho_{2}=3, \varphi_{2}=60^{\circ}(1.04 \mathrm{rad})$ for the second. The intersensor phase-shift corresponding to the DOAs of the sources are $\theta_{1}=-0.18$ and $\theta_{2}=0.58$. The simulations have been performed with an array of $N_{x}=20$ vector sensors recording $N_{t}=128$ time samples each. The $2 \mathrm{C}$ of the original data are represented in Figures $3 \mathrm{a}$ and $3 \mathrm{~b}$. Gaussian noise has been added to a signal-to-noise ratio ${ }^{3} \mathrm{SNR}_{1}=-7 \mathrm{~dB}$ for the first component, and $\mathrm{SNR}_{2}=12 \mathrm{~dB}$ for the second one. To decorrelate the two sources in the interspectral tensor, a frequency-smoothing technique has been used with averaging over five frequency channels. This technique induces

\footnotetext{
${ }^{3} \mathrm{SNR}=$ signal energy on the entire seismic section/noise energy on the entire seismic section.
}

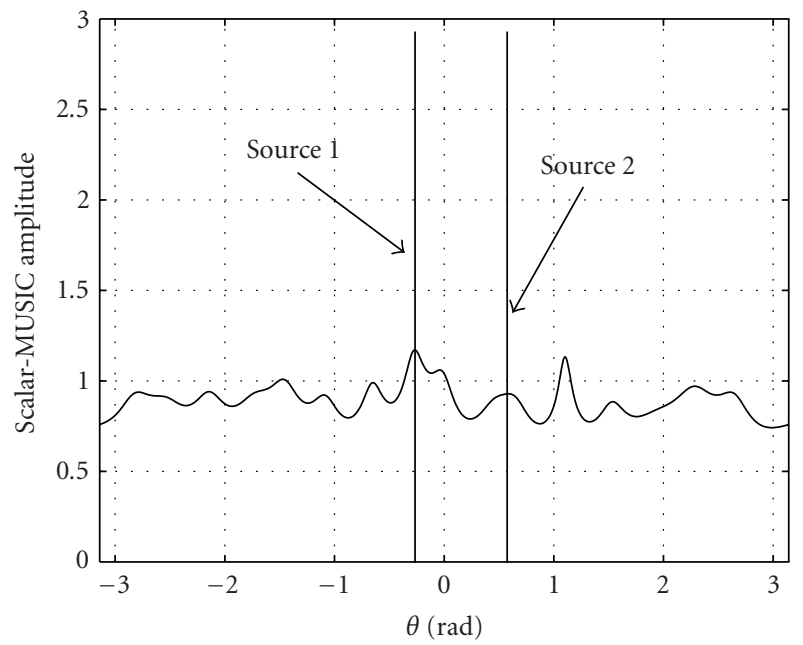

(a)

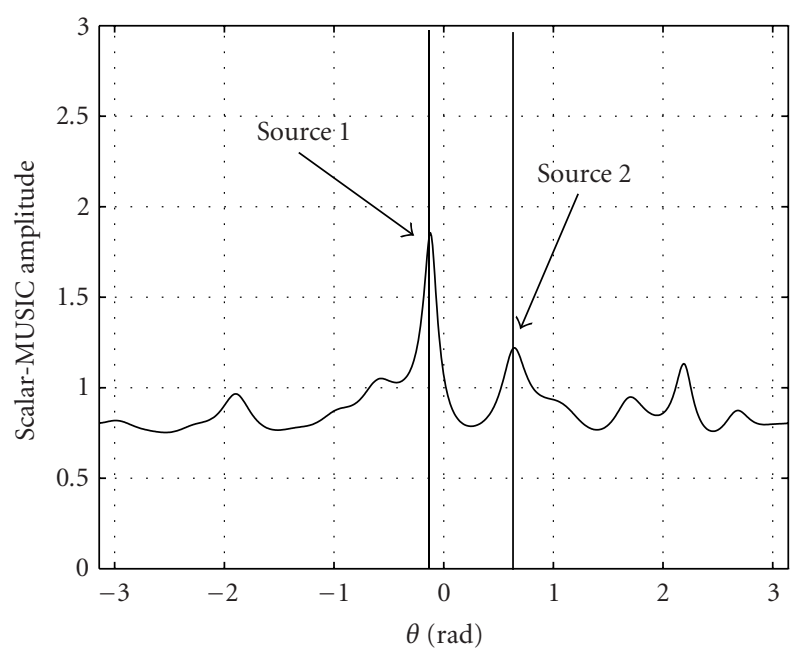

(b)

Figure 4: Scalar MUSIC on each component. (a) On the first component and (b) on the second component.

bias in $\theta$ and $\varphi$ estimates because of the dependency on frequency of these quantities. At the same time, it reinforces the detection of sources having an intersensor phase-shift close to zero, as it is the case for source 1. Vector-MUSIC estimator has been calculated as well as the scalar-MUSIC estimator on each of the components independently. For $\theta$, we have used a computation step of 0.01 in the interval $[-\pi, \pi]$, $\rho \in[0.1: 0.1: 10]$, and $\varphi \in[-\pi: 0.1: \pi]$. In Figures $3 \mathrm{~b}$ and $4 \mathrm{a}$, we have shown the results of the scalar version of MUSIC estimator applied to each component separately. This method is commonly used in seismic vector-sensor array data processing.

It is obvious that, on the first component, the two sources are not clearly visible (Figure 4a). On the second component (Figure $4 \mathrm{~b}$ ), the detection is more accurate but there are still some false alarms that could mask the real detection. 


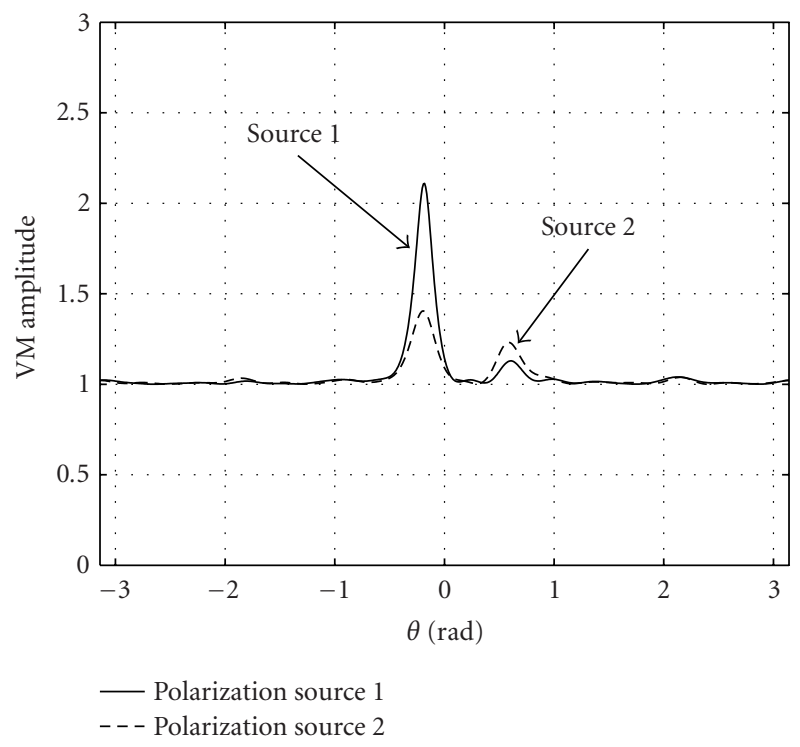

FIGURE 5: Vector MUSIC.

Furthermore, the estimates for $\theta_{1}$ and $\theta_{2}$ are slightly different on the $2 \mathrm{C}$, mainly because of frequency-smoothing technique. Figure 5 shows vector-MUSIC estimator for two sets of polarization parameters. The solid line corresponds to $\rho_{1}=2, \varphi_{1}=-80^{\circ}$ (the polarization parameters of the first source), and the dashed line corresponds to the secondsource polarization parameters $\left(\rho_{2}=3, \varphi_{2}=60^{\circ}\right)$. We can observe (Figure 5) that both detections are a lot smoother than in the scalar case (the secondary lobs are much more attenuated). It means that this estimator is more robust to source correlation than the scalar one, allowing better results for the same averaging technique. This improvement can be explained by the fact that vector-MUSIC algorithm takes into account the relationship existing between the components of vector sensors.

Polarization parameters $\rho$ and $\varphi$ can also be estimated with this method. For two values $\theta_{1}=-0.18$ and $\theta_{2}=0.58$ corresponding to our sources DOAs, we represent the estimator values in the polarization $(\rho ; \varphi)$ plane (Figures $6 \mathrm{a}$ and $6 \mathrm{~b})$. We find the simulated parameters slightly biased due to the frequency averaging.

Vector-MUSIC estimator seems to be less sensitive to source correlation (estimation accuracy) than its scalar version. To illustrate vector-MUSIC sensitivity to spectraltensor estimation precision, we have computed the estimator values for different numbers of statistically independent trials. A scenario with one stationary source corrupted by noise with an SNR $=3 \mathrm{~dB}$ was considered. The intersensor phaseshift corresponding to this source is $\theta \approx 0.49 \mathrm{rad}$ and the polarization parameters are $\rho=2$ and $\varphi=60^{\circ}$. In Figure 7, we have represented the detection lobe corresponding to source polarization parameters, for 10, 100, 500, and 1000 samples. Naturally, the resolution power improves with the number of samples, but the detection is robust even for a poor estimation of spectral tensor (e.g., for 10 samples, the bias in the estimation of $\theta$ is insignificant compared to detection lobe width).

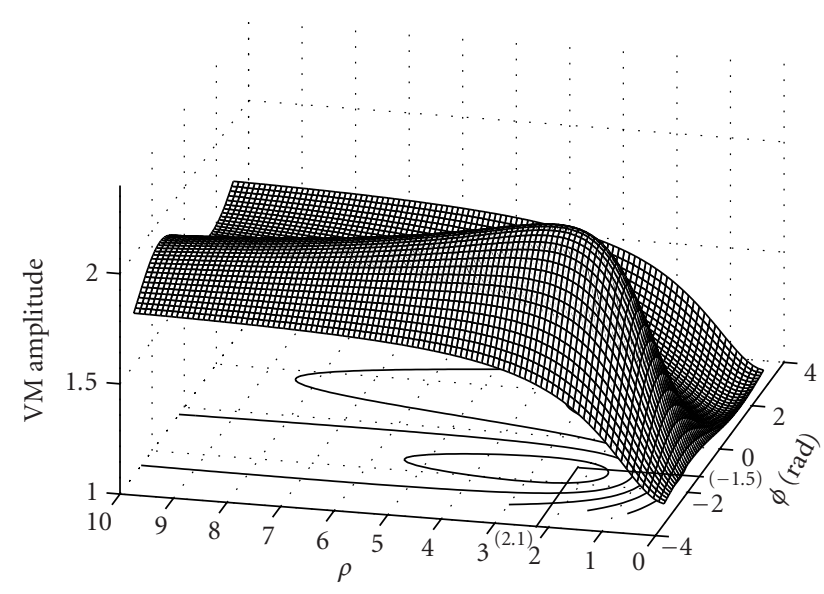

(a)

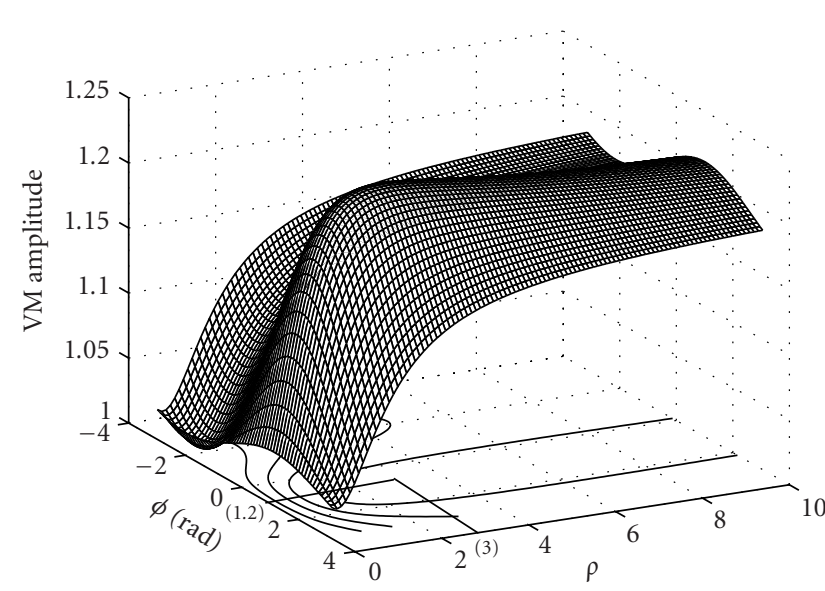

(b)

Figure 6: Vector MUSIC for (a) $\theta=\theta_{1}(-0.18 \mathrm{rad}$ ) and (b) $\theta=$ $\theta_{2}(0.58 \mathrm{rad})$.

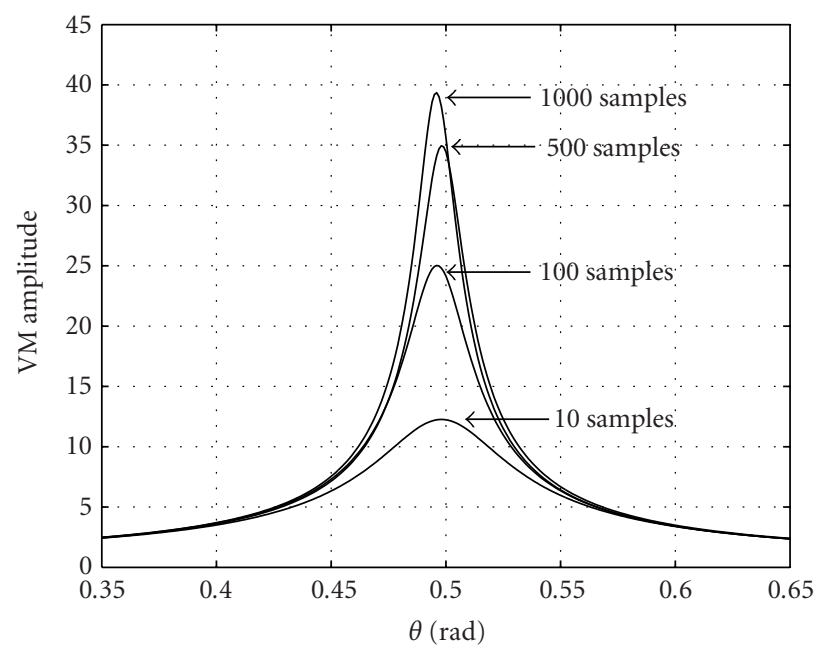

Figure 7: Vector MUSIC for different sample numbers. 


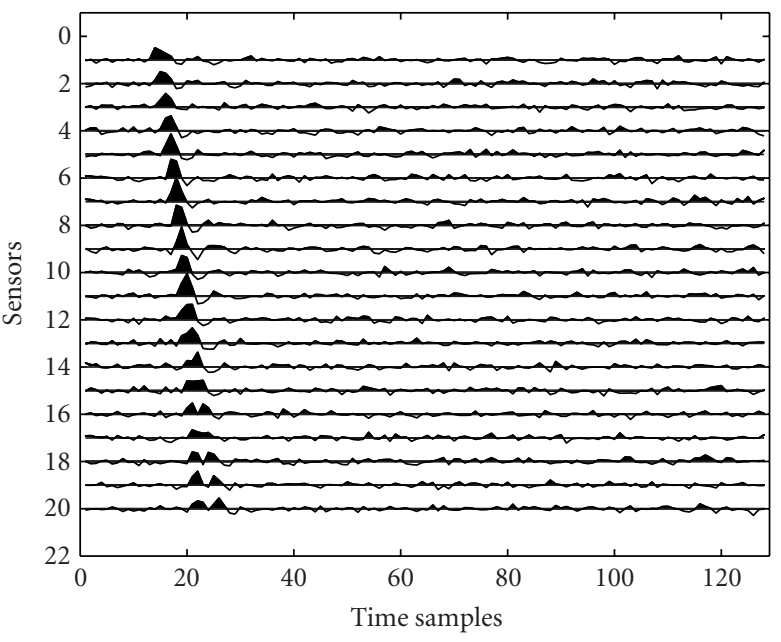

(a)

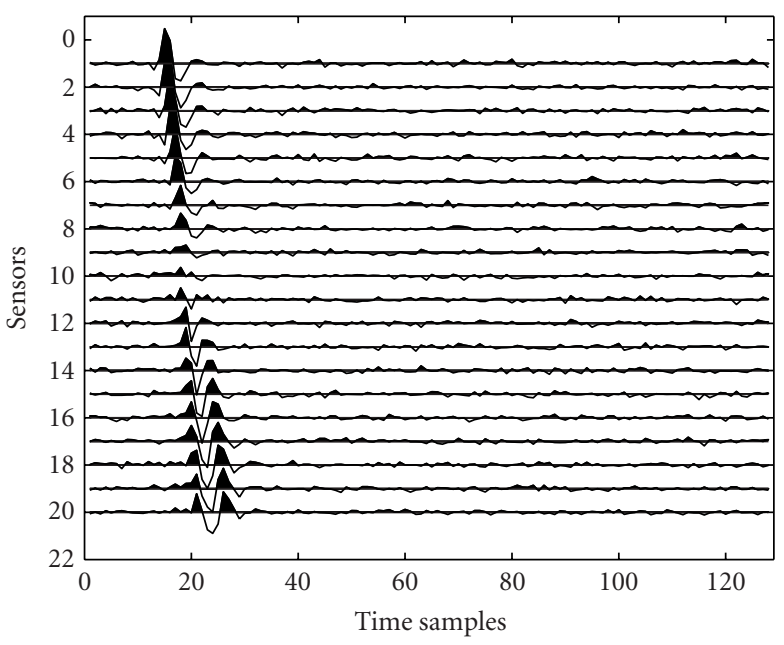

(b)

Figure 8: Two-component seismic section, DOAs closed. (a) First component and (b) second component.

Next, the separation powers of these two algorithms (the scalar and vector MUSIC) will be compared on a synthetic example. We consider the same seismic sources with the same polarization parameters as before $\rho_{1}=2, \varphi_{1}=-80^{\circ}, \rho_{2}=3$, $\varphi_{2}=60^{\circ}$ but with close DOAs (Figures $8 \mathrm{a}$ and $\left.8 \mathrm{~b}\right)\left(\theta_{1}=\right.$ $0.2 \mathrm{rad}$ and $\theta_{2}=0.5 \mathrm{rad}$ ). In this case, averaging over five frequency channels is not an accurate choice because of the important value of the resulting bias. This is why we prefer a mix smoothing technique over three channels in frequency, and three sensors along the antenna (spatial smoothing). The results of MUSIC algorithm applied on each component independently is shown in Figures 9a and 9b. The theoretical position of the detection peaks has been marked by a vertical line for each source. One can see that on the first component, scalar-MUSIC estimator presents only one detection peak corresponding to an average DOA of the two sources.

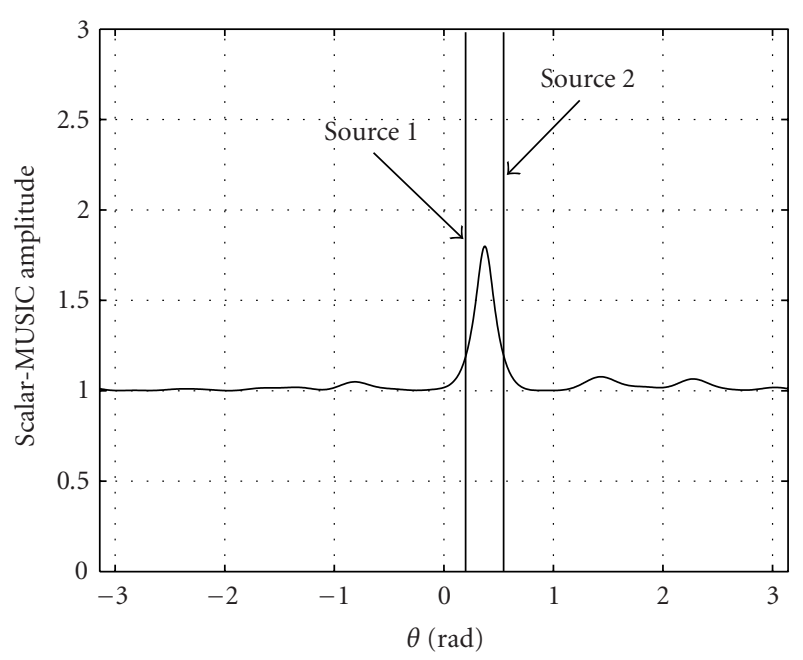

(a)

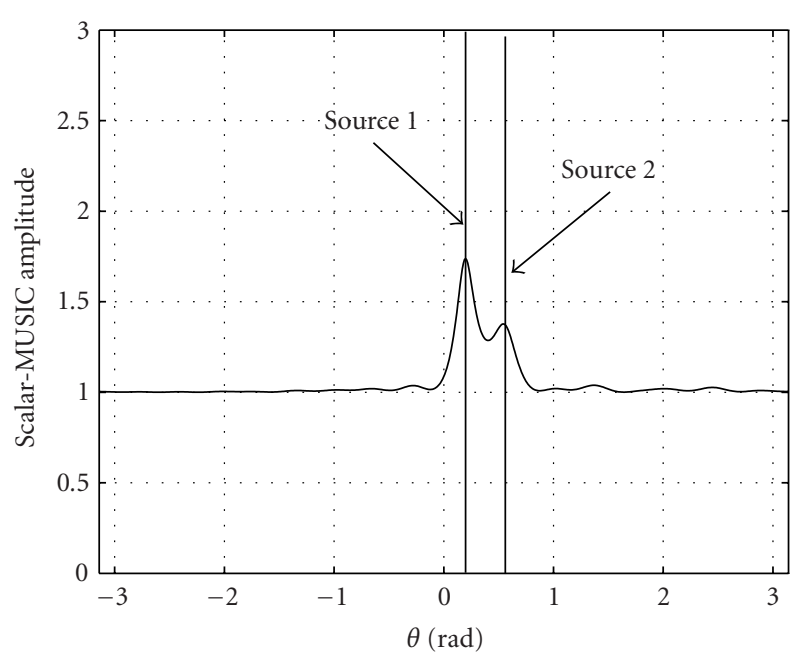

(b)

Figure 9: Scalar MUSIC on each of the components separately. (a) On the first component and (b) on the second component.

On the second one, the sources are correctly detected but the detection lobes are partially superposed. Vector-MUSIC curves, plotted for polarization parameters corresponding to the incoming waves, give a more accurate DOAs characterization, isolating each source (Figure 10). If we continue to bring $\theta_{1}$ closer to $\theta_{2},\left(\theta_{1}=0.3 \mathrm{rad}, \theta_{2}=0.5 \mathrm{rad}\right)$, the scalar version of the algorithm fails to detect both sources, even on the second component (Figure 11), while the multicomponent version still performs a correct detection (Figure 12). The explanation is on one side, the use of coherent information between the $2 \mathrm{C}$, and on the other side, the isolation of each source on one detection curve corresponding to its parameters of polarization. This way, the superposition of detection lobes is avoided.

This algorithm performs an averaging between the $N_{c}$ components, so, it should be used only when the SNR is 


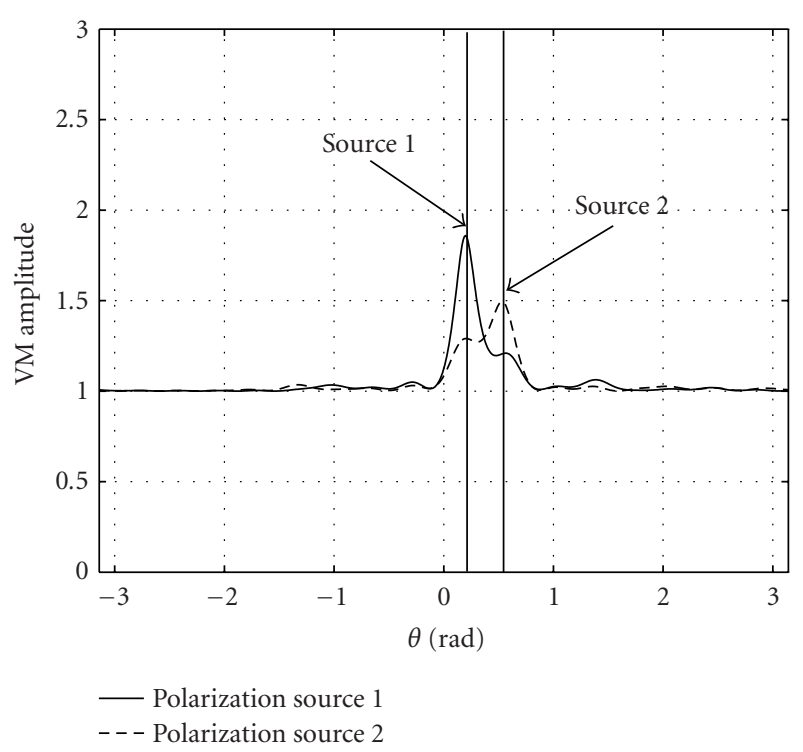

FIgURE 10: Vector MUSIC for the 2C dataset presented in Figure 8.

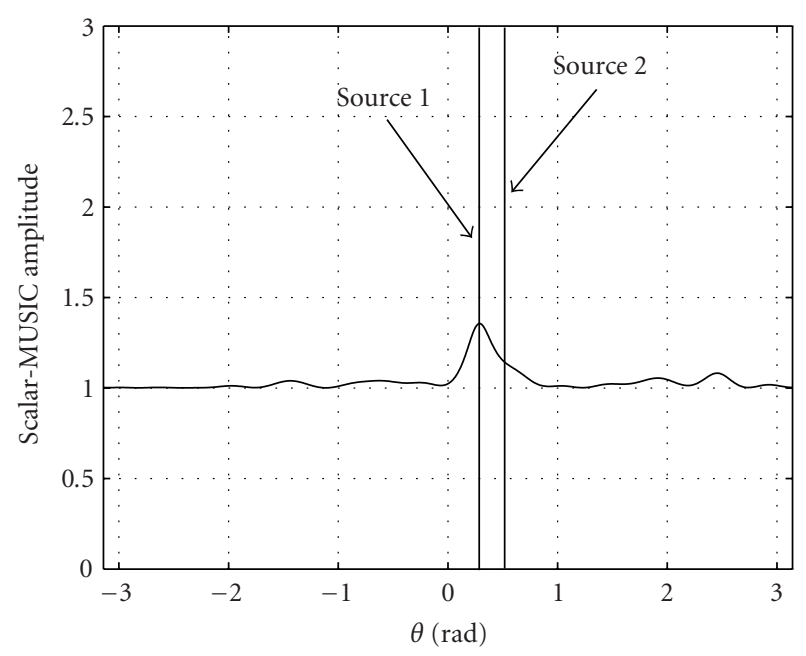

FIGURE 11: Scalar MUSIC on the second component for very close DOAs.

comparable on all of them. Using a very noisy component could strongly deteriorate the estimation performance of the algorithm. Also, the detection of sources having exactly the same DOA is theoretically impossible, even if they have different polarizations. In this case the algorithm would estimate one wave with polarization parameters equal to the mean of the impinging waves.

Figure 13 plots the DOA root mean square (RMS) estimation error for the proposed algorithm and the scalar version. Two equal-power uncorrelated polarized sources with random initial phases impinge on a $2 \mathrm{C}$ sensor-array com-

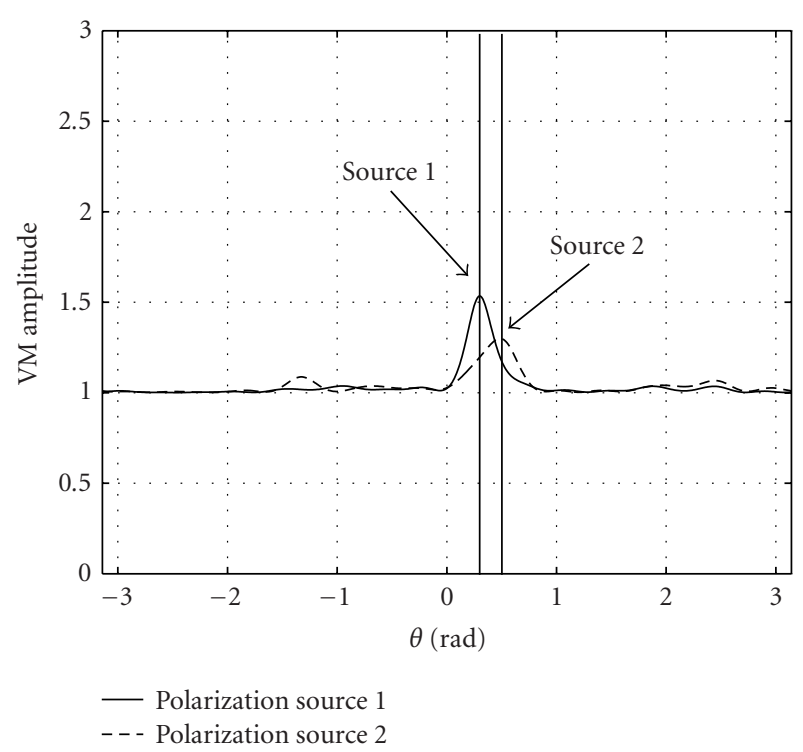

FIgURE 12: Vector MUSIC for very close DOAs.

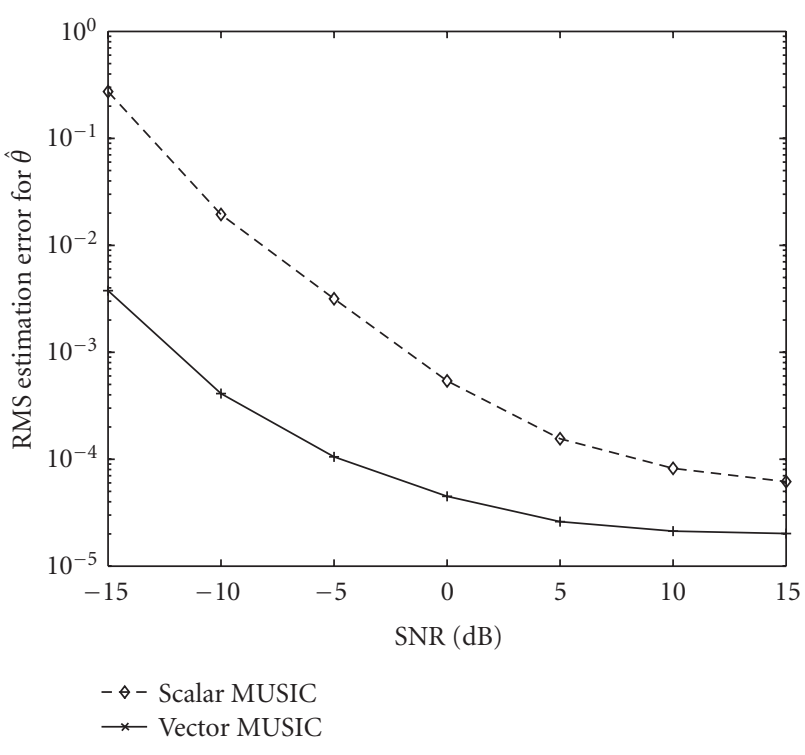

FIGURE 13: RMS estimation error of $\hat{\theta}$.

posed of 10 identical, equally spaced sensors. The sources DOAs are $\theta_{1}=-5^{\circ}, \theta_{2}=10^{\circ}$, and they have the following polarization parameters: $\rho_{1}=2, \varphi_{1}=-15^{\circ}, \rho_{2}=3$, $\varphi_{1}=20^{\circ}$. One hundred frequency samples are used in each Monte Carlo simulation run. Five hundred independent Monte Carlo runs contribute to each data point on each figure. Additive white Gaussian noise (AWGN) is present, and the SNR figures are calculated relative to each signal power. The RMS error of $\hat{\theta}$ plotted in these figures is defined as the RMS of the RMS estimation error of $\hat{\theta}_{1}$ and $\hat{\theta}_{2}$. For scalar MUSIC, an averaging is operated over the $2 \mathrm{C}$. 
Figure 13 shows that the proposed vector MUSIC algorithm yields better results than its scalar version improving estimator behavior, especially for low SNR.

\section{CONCLUSIONS}

Polarization provides an additional dimension to source parameters space. Taking it into account, we have proposed a new model and an eigenstructure-based algorithm based on the intrinsic data structure that enables characterization of $N_{c}$ times more sources than in the monocomponent array case. Simulations were carried out to evaluate the performance of the proposed method. We have shown that polarization diversity enhances the performance of the direction finding system. Both, accuracy and resolving power are improved. Vector-MUSIC algorithm is less sensible to the correlation of the sources than its scalar version, providing at the same time a better separation power. The algorithm is very flexible, allowing the choice of the number of parameters to estimate. This permits the extension of vector-MUSIC algorithm to an arbitrary number of components of a vectorsensor array.

\section{REFERENCES}

[1] R. E. Sheriff and P. G. Lloyd, Exploration Seismology, Cambridge University Press, Cambridge, UK, 2nd edition, 1995.

[2] R. O. Schmidt, "Multiple emitter location and signal parameter estimation," IEEE Trans. Antennas and Propagation, vol. 34, no. 3, pp. 276-280, 1986.

[3] A. Nehorai and E. Paldi, "Vector-sensor array processing for electromagnetic source localization," IEEE Trans. Signal Processing, vol. 42, no. 2, pp. 376-398, 1994.

[4] J. Li and R. T. Compton Jr., "Angle and polarization estimation using ESPRIT with a polarization sensitive array," IEEE Trans. Antennas and Propagation, vol. 39, no. 9, pp. 1376 1383, 1991.

[5] K. T. Wong and M. D. Zoltowski, "Self-initiating MUSICbased direction finding and polarization estimation in spatiopolarizational beamspace," IEEE Trans. Antennas and Propagation, vol. 48, no. 8, pp. 1235-1245, 2000.

[6] K. T. Wong and M. D. Zoltowski, "Diversely polarized rootMUSIC for azimuth-elevation angle-of-arrival estimation," in Proc. IEEE Antennas and Propagation Society International Symposium (APSIS '98), vol. 2, pp. 1352-1355, Baltimore, Md, USA, July 1996.

[7] K. T. Wong and M. D. Zoltowski, "Root-music-based azimuth-elevation angle-of-arrival estimation with uniformly spaced but arbitrarily oriented velocity hydrophones," IEEE Trans. Signal Processing, vol. 47, no. 12, pp. 3250-3260, 1999.

[8] K. T. Wong and M. D. Zoltowski, "Uni-vector-sensor ESPRIT for multisource azimuth, elevation and polarization estimation," IEEE Trans. Antennas and Propagation, vol. 45, no. 10, pp. 1467-1474, 1997.

[9] M. D. Zoltowski and K. T. Wong, "Closed-form eigenstructure-based direction finding using arbitrary but identical subarrays on a sparse uniform Cartesian array grid," IEEE Trans. Signal Processing, vol. 48, no. 8, pp. 2205-2210, 2000.

[10] K. T. Wong, "Blind beamforming/geolocation for widebandFFHs with unknown hop-sequences," IEEE Trans. on Aerospace and Electronics Systems, vol. 37, no. 1, pp. 65-67, 2001.

[11] M. D. Zoltowski and K. T. Wong, "ESPRIT-based 2-D direction finding with a sparse uniform array of electromagnetic vector sensors,” IEEE Trans. Signal Processing, vol. 48, no. 8, pp. 2195-2204, 2000.

[12] K. T. Wong and M. D. Zoltowski, "Closed-form direction finding and polarization estimation with arbitrarily spaced electromagnetic vector-sensors at unknown locations," IEEE Trans. Antennas and Propagation, vol. 48, no. 5, pp. 671-681, 2000.

[13] H. Akaike, "A new look at the statistical model identification," IEEE Trans. Automatic Control, vol. 19, no. 6, pp. 716-723, 1974.

[14] G. Bienvenu and L. Kopp, "Principe de la goniométrie passive adaptative," in Proc. 7'eme Colloque sur le Traitement du Signal et ses Applications (GRETSI '79), pp. 106/1-106/10, Nice, France, 1979.

[15] J. E. Evans, J. R. Johnson, and D. F. Sun, "High resolution angular spectrum estimation techniques for terrain scattering analysis and angle of arrival estimation," in Proc. 1st ASSP Workshop Spectral Estimation, pp. 134-139, Hamilton, Ontario, Canada, August 1981.

[16] L. C. Godara, "Beamforming in the presence of correlated arrivals using structured correlation matrix," IEEE Trans. Acoustics, Speech, and Signal Processing, vol. 38, no. 1, pp. 1-15, 1990.

[17] N. Thirion, J.-L. Lacoume, and J. Mars, "Resolving power of spectral matrix filtering: A discussion on the links steering vectors/eigenvectors," in Proc. 8th IEEE Signal Processing Workshop on Statistical Signal and Array Processing (SSAP '96), pp. 340-343, Corfu, Greece, June 1996.

[18] J. Mars, F. Glangeaud, J. L. Lacoume, J. M. Fourmann, and S. Spitz, "Separation of seismic waves," in Proc. 56th Meeting of Society of Exploration Geophysicists, pp. 489-492, New Orleans, La, USA, 1987.

[19] S. Anderson and A. Nehorai, "Analysis of a polarized seismic wave model," IEEE Trans. Signal Processing, vol. 44, no. 2, pp. 379-386, 1996.

[20] T. G. Kolda, "Orthogonal tensor decompositions," SIAM Journal on Matrix Analysis and Applications, vol. 23, no. 1, pp. 243-255, 2001.

[21] L. De Lathauwer, Signal processing based on multilinear algebra, Ph.D. thesis, Katholieke Universiteit Leuven, Leuven, Belgium, 1997.

Sebastian Miron was born in Suceava, Romania, in 1977. He graduated from Universitatea Technicǎ "Gh. Asachi” Iasi, in 2001, and received his M.S. degree in signal, image, and speech processing from the Institut National Polytechnique de Grenoble, France, in 2002. He is currently pursuing his Ph.D. degree at the Laboratoire des Images et des Signaux, Grenoble, France. His current research interests include seismic data processing, multilinear algebra, and quaternions.

Nicolas Le Bihan was born in Morlaix, France, in 1974. He obtained his B.S. degree in physics from Université de Bretagne Occidentale (UBO), Brest, in 1997. He received his M.S. and Ph.D. degrees in signal processing, respectively, in 1998 and 2001, both from the Institut National Polytechnique de Grenoble (INPG), Grenoble. Since 2002, he is Chargé de Recherche at the Centre National de la Recherche Scientifique (CNRS) and is working in the Laboratoire des Images
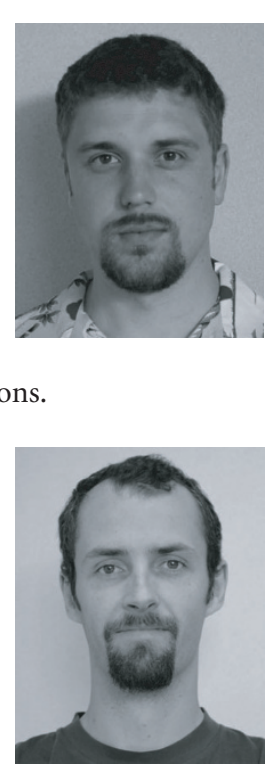
et des Signaux (UMR 5083), Grenoble, France. His research interests include polarized signal processing using multilinear algebra and hypercomplex numbers techniques, and applications of signal processing in geophysics.

Jérôme I. Mars received his M.S. degree in 1986 in geophysics from Joseph Fourier University, Grenoble, France, and his Ph.D. degree in signal processing in 1988 from the Institut National Polytechnique de Grenoble, France. From 1989 to 1992, he was a Postdoctoral Researcher at the Centre d'Etudes des Phénomènes Aléatoires et Géophysiques, Grenoble, France. From 1992 to 1995, he was a Visiting Lecturer and

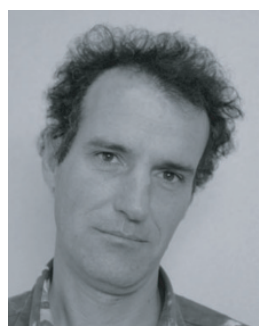
Scientist in the Materials Sciences and Mineral Engineering Department, University of California, Berkeley. He is currently an Associate Professor of signal processing at the Institut National Polytechnique de Grenoble, and is with the Laboratoire des Images et Signaux, Grenoble, France. His research interests include seismic and acoustic signal processing, wavefield separation methods, time-frequency time-scale characterization, and applied geophysics. He is a Member of the Society of Exploration Geophysicists (SEG) and European Association of Geoscientists and Engineers (EAGE). 